\title{
How Bee-like Agents Support Cultural Heritage
}

\author{
Martí Fàbregas, Beatriz López, and Josep Masana \\ University of Girona, Campus Montilivi, edifici P4, 17071 Girona \\ \{marti, blopez\}@eia.udg.es \\ http://www.iiia.udg.es
}

\begin{abstract}
Elderly people are a great repository of knowledge, the majority of which has never been gathered by formal means. In this paper we introduce an application of multi-agent systems to support knowledge acquisition from this rich repository knowledge which is only available from elderly and experienced people. Our system provides the opportunity to complement different versions of the same knowledge produced in an extensive geographical and cultural region with the main objective of supporting Cultural Heritage. Users without much technological knowledge can search or leave information about some type of knowledge. Then, the system behaves like a swarm of bees, in this way the bee-like agents process the user contributions and the knowledge emerges from the system. Queen-like agents, honey-bee, drones and foragers have different roles inside the hive: looking for information resemblances, computing information confidence, checking the necessity of knowledge validation, and updating user's reliability. The system's feasibility has been tested on the specific area of ethnobotany, which concerns the ways in which specific societies name and classify plants.
\end{abstract}

\section{Introduction}

The speed at which the Internet is evolving can hide several questions related to our culture and traditions. Efforts in computer science in general, and agent technology in particular, are usually aimed at improving technology, so new capabilities are added to future systems. Most of the systems rely on formal knowledge, which is the main component of the systems. However we should be aware that most of our current traditional knowledge can disappear in the near future. We are not referring to formal forms of knowledge, but to the knowledge that only elderly people know.

As the Valencian writer Bernat Capó said: "When an elderly farmer dies it is as if a small library has burnt down" [4].

Elderly people are a great repository of knowledge the majority of which has never been gathered formally. Current technology has arrived to almost everywhere: city suburbs and social centers of any population have an access point to the net. However, the net is being used to bring information from new knowledge producers to the general public, and there is no flow in the opposite direction, that is, gathering and verifying information that people have and supplying it to the providers. 
There are several examples of information gathering in specific knowledge fields. For example, in Spain, several authors have been supported by private and public local companies, for instance Joan Pellicer and his "Costumari botanic" [10]. This kind of work has been achieved by means of traditional field work, in which most people are involved in a manual process and the results are achieved in the long term. Our aim is to provide an alternative method to favor the conservation of a certain kind of information (traditional, coming from people) by means of the new technologies, and particularly, with the use of agent technology. Moreover, by gathering traditional knowledge in an automated way our system gives the opportunity to complement different versions of the same knowledge produced in an extensive geographical and cultural region.

In this paper we explain our multi-agent system En_C_Prou [1] where this traditional knowledge acquisition is performed. Our system is based on the paradigm of Swarm intelligence. Users without much technological knowledge can search or leave information about some type of knowledge. Then, the system behaves like a swarm, in such a way that bee-like agents process the user contributions and the knowledge emerges from the system. Queen-like agents, honey-bees, drones and foragers have different roles inside the hive: looking for information resemblances, computing information confidence, checking the necessity to validate knowledge, and updating user's reliability. The system's feasibility is being tested on the specific area of ethnobotany, which is concerned with the ways in which specific societies name and classify plants [10].

This paper is organized as follows. First, we introduce our approach to beelike agents in section 2. Then we explain how the knowledge emerge from the swarm in section 3 . We continue by shown our current experimental results in section 4 . In section 5 we frame our system in previous works. And we end in section 6 with several conclusions and discussion.

\section{Swarm-like multi-agent system}

Swarm Intelligence is the name of a line of research with a lot of potential in the field of Artificial Intelligence [3,2]. Swarm Intelligence refers to the capacity to solve complex problems with simple interactions. Insect colonies function in the following manner: without general supervision they undertake distributed work where each individual is concerned with a part of the work. From the work of all the individuals the solutions to complex problems emerge.

Most researchers have adopted the metaphor of the colony's social behavior to build artificial multi-agent systems. Thus complex behaviors are supported by simple agents, either in mass, time or scope [16]. Multi-agent systems based on swarm intelligence are self-organized systems that require interactions between agents (insects): direct interactions and indirect interactions. Direct interactions are agent-to-agent interactions, while indirect interactions are agentto-environment interactions, usually known as stigmergy [5,2]. Two individuals interact indirectly when one of them modifies the environment and the other responds to the new environment at a later time. 
We have taken this self-organization approach of multiagent systems to model our traditional knowledge gathering system. This approach enables knowledge to emerge from the information provided by a myriad of different and diverse users.

In particular, we adopt the model of swarms of bees to implement the En_C_Prou system, so that each individual in the hive (working bees, drones, queens, foragers and honey bees) is represented by an agent, while users are beekeepers (see Figure 1).

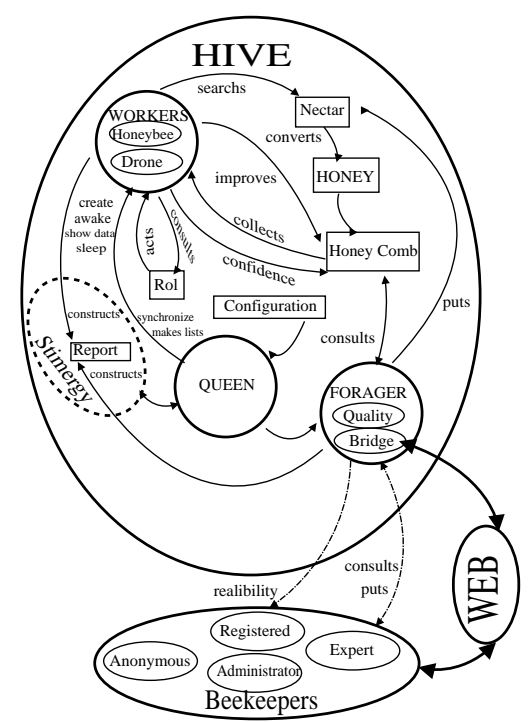

Fig. 1. Individual agents and their interaction in the EnCProu system.

\subsection{En_C_Prou general view}

En_C_Prou is mainly concerned with the web interface and the hive. Users (beekeepers) access a web page where they can either carry out their consultations or make contributions. The user has a menu from which he/she can choose from among different actions: making a contribution to the subjects in the system, entering a new concept, creating a new attribute, querying a particular subject, validating the knowledge of the system, etc. Each action is enabled according to the user's reliability degree, which is represented by a numerical value in $[0,1]$. Depending on the chosen action, the user is guided step by step through other web pages in order to carry out the activity selected with the mouse. So the system has an easy interface accessible to non technological users, as elderly people usually is. The contents of the web pages are dynamic and represent the current information in the system. 
For the sake of simplicity, in this paper we focus on the multi-agent system supporting the hive. The swarm processes information provided by users in the context of a traditional knowledge area, and keeps it in different honey combs. So from particular user contributions (nectar), bee-like agents produce knowledge (honey) that is kept in honey combs. At the end, users acting as beekeeper can access this knowledge.

The system always takes it for granted that beekeepers are honest when providing the information. However, it is necessary to note, that the knowledge contained in the system is not strictly academic, and therefore, it should be interpreted with the confidence that corresponds to any type of traditional knowledge. The model supposes that a user can have certain information on a subject but that he/she has not been able to relate this information with another subject with important degrees of coincidence because, for example, the terminology used by another user is different. Then, the system searches for similarities between the different information entered by the users in order to infer knowledge from all the information received. Moreover, the system keeps links between similar concepts to make cross-referencing knowledge easier. This makes accessing the system's knowledge easier for the user, saving time in revising information given by other users, favoring the incorporation of information, and allowing the final users to consult the knowledge kept in the hive quickly. This knowledge is validated a posteriori by the expert beekeepers. This validation process is performed periodically, depending on the amount of contributions provided by users and their reliability.

It is important to note, that user' reliability evolves according to the usersystem interaction. Users with a low reliability can become experts if the system proves that their contributions have been useful to the swarm.

\section{$2.2 \quad$ Bee-like agents}

Agents build and maintain the honey combs, the spatiostructure regarding the information that they collect. That is, each honey comb contains knowledge related to a single concept in the application. For example, in the case of ethnobotany, each honey comb is a plant and the hive represents the complete knowledge of the application.

There are three main kinds of bees that are implemented as agents: the queen, workers and foragers. The queen is responsible for the brood, for keeping the hive with enough bees to deal with the amount of information currently available in the system. Workers, as suggested by their name, carry out the work related to converting information into knowledge. There are two main types of workers: drones and honey bees. Drones fertilize the information, in two ways:

1. First, they are aware of the quality and amount of information in the system, so they influence the activity of the hive in order to validate the information.

2. Second, they look for similarities between the different honey combs, so that the information can be cross-referenced (fertilization) and thus the knowledge in the hive enriched. 
The honey bees are in charge of gathering information about a single concept. They get user information (nectar) and bring it up to the cells in the form of honey by using previous contributions of other users.

Finally, foragers are in charge of finding nectar sources. They have two main roles: Firstly, as bridge-foragers with the external environment, so they are aware of the current users in the system and their most recent contributions. Secondly, as quality-foragers, keeping information about the users' reliability.

\subsection{Coordination}

Coordination of the different agents is mainly achieved by indirect communication, that is, with the use of stigmergy mechanisms. Initially, beekeepers introduce information about a subject (plants) from the web page environment. Bridge foragers are aware of the information that the users are registering and they report such new information (nectar) on the information board of the hive.

Each worker decides its role (honey bee or drone) depending on the state of the hive. A honey bee enters the data introduced by the beekeeper (nectar) and converts it into knowledge (honey). To carry out this task, the honey bee searches in the immediate references provided by the foragers. Then it computes the confidence values of the contributions.

When the honey bee finishes its work it reports to the swarm about its performance. Then, with this report honey bees related to the new knowledge wake up. At the same time a drone finds out if there is information that needs to be verified as a consequence of the new knowledge incorporated into the system.

The drone reports the need to check the knowledge related to a single subject of the system together with the revision data. The drone is also the bee-like agent responsible for providing information about the concepts that have a high degree of coincidence (resemblance).

According to the drone's report about the need to check the knowledge, beekeepers validate the system's knowledge. External knowledge validation performed by users assures, to some extend, that the knowledge obtained by the swarm has a certain degree of quality.

A quality forager agent then revises the reliability degree of each user related to the validated knowledge. Users involved in the contributions that have converged on validated knowledge can increase his/her reliability; conversely, the user's reliability can also be decreased. If the reliability of a given user changes, the honey comb status depending on his/her information should be revised by the corresponding honey bees.

Reports and nectar are the stigma used for agent coordination. Occasionally, direct communication is also used between the queen and other agents. For example, when the queen creates a honey bee or drone.

To illustrate the complete system let us apply it in an ethnobotany context. In a given moment of time, the hive includes a honey comb related to the plant thyme (the concept). The knowledge stored in the honey comb is that thyme is a culinary and medical plant. The confidence of this information is 0.75 and 0.3. Moreover, there are some relationships with other plants (honey combs): 
fennel and oregano. A registered user with a 0.3 degree of reliability enters new information about thyme. In particular, the user says that the color of the thyme flower is violet. Bridge foragers bring this information as a report to the nectar repository. Then, honey bees related to thyme wake up, and convert the information "The color of the thyme flower is violet" to the knowledge "The color of the thyme flower is violet, confidence 0.3 " at the honey comb. Now, a drone looks for similar flower colors in the hive and makes a report. Honey bees concerned with the report wake up and check the information contained in the thyme honey comb in order to improve its knowledge, and so on. The queen bee plays her role when the users enter information related to a new plant. Then she is in charge of creating a new honey bee that will start a new honey comb.

\subsection{Hive}

The hive is the database in which all the system's knowledge is stored. Each individual concept of knowledge is represented in a honey comb. Links between related concepts are established as well as links between the different honey combs. Each honey comb can have a different number of attributes. An attribute corresponds to a user contribution that is processed and transformed into knowledge, and stored in a cell of the honey comb. The initial set of attributes available to describe concepts is predefined. Then, honey combs are modeled according to the different contributions that the users provide. Each honeycomb contains cells that collect the contributions about attributes, together with the confidence of the data contained in them (see Figure 2).

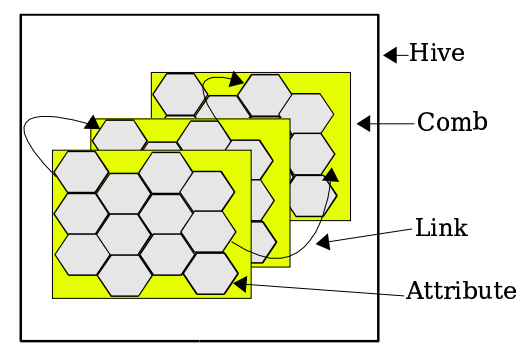

Fig. 2. Visual representation of the system's data base (hive).

Confidence of an attribute is related to a value in the interval $[-1,1]$. This confidence expresses the reliability of the user or the set of users that have provided the contribution, as well as similar information in the system that supports the knowledge. A negative value indicates that the user denies the attribute with his/her contribution, consequently the attribute's confidence level is the negative of the user's reliability. If the confidence value is positive, it means that the user confirms the attribute as a characteristic of the subject, and the attribute take the same confidence degree as the user's reliability. For example, 
in the ethnobotany application we can obtain the "aromatic" attribute for the laurel plant with the following contributions:

- An anonymous user with reliability 0.1 gives a positive opinion. Confidence contribution $=0.1$

- A registered user with reliability 0.3 gives a negative opinion. Confidence contribution $=-0.3$

- An expert user with reliability 0.8 gives a positive opinion. Confidence contribution $=0.8$

The confidence levels of the users' contributions to an attribute is combined to determine a final confidence degree, as explained in the following section.

\section{$3 \quad$ Emerging knowledge}

From the contributions made by the users the swarm acts, so we can say that the system infers knowledge from data. This process is not a centralized and sequential process, but the knowledge emerges as a consequence of the individual activities carried out by the agents in the system according to the following methods:

- The honey resemblance method, based on similarity measures

- The information confidence method, based on MYCIN certainty factors

- The degree of necessity to check information method, based on fuzzy logic

- The reliability update method, based on fuzzy logic too.

Figure 3 shows the difference between a system that gathers information and the knowledge kept in the En_C_Prou system.

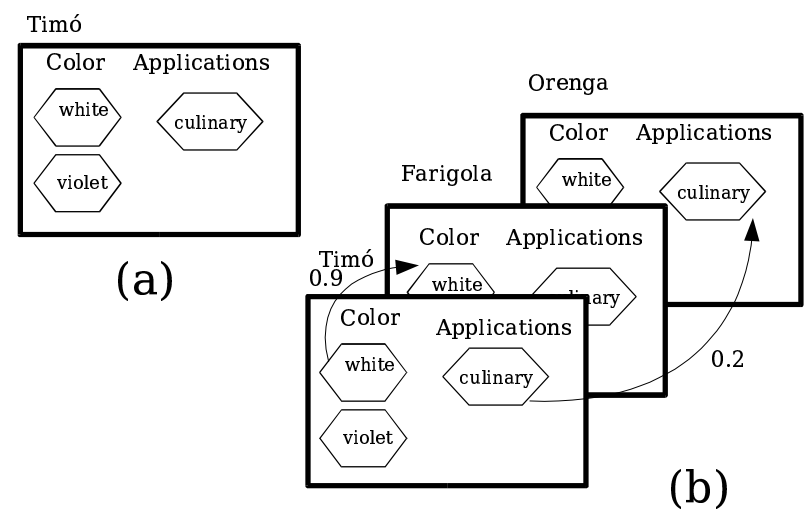

Fig. 3. (a) A collection of data. (b) The results of processing the information with En_C_Prou.

In the next subsections the methods deployed by each bee-like agent are explained. Namely, the honey comb resemblance, the information confidence, the degree of necessity to check information, and the reliability update methods. 


\subsection{Resemblance of honeycombs}

One of the most valuable functions that the system has for the user is that it compares honeycombs. In the case of ethnobotany comparing the honeycombs establishes resemblances between different plants. These honey comb comparisons allow the drone to build links between different honey combs, so the knowledge from a honey comb is related to and supported by knowledge in other honey combs.

In artificial intelligence, resemblance methods are often called similarity methods. However, it is important to note that we are not looking for identical pieces of knowledge but for resemblances that allow the inference of knowledge to be supported.

There are many research papers by many researchers on similarity measures [17]. However, there is no universal guide on which method is most suitable. The choice of method depends a lot on the application domain.

Thus, for the particular case of ethnobotany, it is very complicated to find a single similarity criterion between plants. For one person, a plant A can be similar to a plant B because B performs a similar function, while for another person, the resemblance is due to the habitat that both plants share. The field of ethnobotany is then a clear example for understanding the implicit complications that the treatment of traditional knowledge can entail. Finally, after several studies, we have determined that two plants are similar according to the attributes that they share. This has been formalized in the following methodology.

Given two plants P1 and P2, the following measures are performed: the number of common attributes (\#ccom), the union of attributes (\#ctot), and the number of attributes with the same value in both plants (\#ceq). Then, the resemblance between $\mathrm{P} 1$ and $\mathrm{P} 2$ is determined as follow:

$$
R(P 1, P 2)=\alpha * \operatorname{sim}_{y} 1(P 1, P 2)+\beta * \operatorname{sim}_{2}(P 1, P 2)
$$

where

$$
\begin{aligned}
& \operatorname{sim}_{1}(P 1, P 2)=\frac{\# \text { ceq }}{\# \text { ccom }} \\
& \operatorname{sim}_{2}(P 1, P 2)=\frac{\# \text { ccom }}{\# \text { ctot }}
\end{aligned}
$$

In particular, we have experimentally chosen $\alpha=\beta=0.5$. Table 3.1 shows an example of the application of the measure.

\subsection{Knowledge confidence}

The information that remains as knowledge in the hive is the combination of all the contributions given by the users. Remember that each contribution is accompanied by a confidence value in $[-1,1]$. This confidence value corresponds to the reliability of the user that has provided the contribution related to the attribute. However, if there are several users that have made different contributions then the attribute stored in the hive should have a combined confidence valued. There 


\begin{tabular}{|l|l|l|l|l|l|l|l|}
\hline P1 & P2 & \#ccom & \#ctot & \#ceq & sim $_{1}$ & sim $_{2}$ & $\mathrm{R}$ \\
\hline 6 & 10 & 4 & 8 & 3 & 0.75 & 0.5 & 0.63 \\
\hline 16 & 20 & 4 & 28 & 3 & 0.75 & 0.14 & 0.45 \\
\hline 1 & 1 & 1 & 1 & 1 & 1 & 1 & 1 \\
\hline 4 & 10 & 4 & 6 & 3 & 0.75 & 0.67 & 0.71 \\
\hline
\end{tabular}

Table 1. Examples of resemblances between different plants, P1 and P2. In columns corresponding to P1 and P2 the total number of attributes of each plant is shown.

are different methods of combining these values [15]. However, the process we are interested in should be incremental, since knowledge confidence should be modified as new contributions arrive. A suitable and very well known method of propagating evidence is the MYCIN certainty factors method [13] that we have chosen because of its simplicity.

Given a set of user contributions about an attribute of a subject, the confidence value of the attribute is computed according to the following procedure:

1. Contributions are sorted according to their absolute value.

2. The certainty factor combination formulas are then applied.Given two contributions $\mathrm{x}$ and $\mathrm{y}$, with their corresponding confidence values $c_{x}, c_{y}$,

(a) If both contributions confirm the attribute $\left(c_{x}>0, c_{y}>0\right)$ : Resulting confidence $=c_{x}+c_{y}-c_{x} * c_{y}$

(b) If both contributions deny the attribute $\left(c_{x}<0, c_{y}<0\right)$ : Resulting confidence $=c_{x}+c_{y}+c_{x} * c_{y}$

(c) If there are discrepancies between the informers $\left(c_{x}>0, c_{y}<0\right.$ or $\left.c_{x}<0, c_{y}>0\right)$ : Resulting confidence $=c_{x}+c_{y}$

Note, that this method is not incremental, because all the data is stored in the system. So each time a new contribution arrives, the first step is applied with all previous values.

To illustrate the method with an example, let's suppose that the following contributions are available for the subject laurel: Aromatic with the following confidence values: $0.1,0.8,-0.3$. Note that contributions with a positive value are confirming the attribute, while contributions with a negative value are denying it. After applying the first step the resulting order (with absolute values) of contributions is: $0.1,-0.3,0.8$. Then, the first combination is carried out between 0.1 and -0.3 . Since both contributions are divergent it is necessary to apply case (c), the outcome of which is -0.2 . The next step is to combine this result -0.2 with the 0.8 evidence. These contributions are also divergent, case (c) is applied again, obtaining the final confidence value of 0.6 for the Aromatic attribute of the Laurel concept. As the reader can observe, the contribution provided by the user with highest reliability (0.8) is the one that has the most influence in the final result. For this reason, we are currently exploring alternative methods [14]. 


\subsection{The degree of necessity to check information}

In the previous subsection, we described how honey bee agents decide about the confidence of an attribute from the contributions of the users. However, confidence of an attribute also depends on the number of contributions. That is, it is not the same to have a confidence value of 0.4 from 2 contributions as having a confidence value of 0.4 from 40 contributions. It is natural to think that in the second case it is necessary that an expert verifies the information. Two contributions are very few, and it is not necessary to burden a beekeeper with validating this knowledge. However, with more than 40 contributions we can say that there is a clear diversity of opinion and the approval of an expert is very necessary. Drones are responsible for monitoring this condition in relation to the knowledge stored in the hive and computing the degree of necessity to validate the knowledge.

The need to check is an imprecise concept, there is a "high" need to check, or a "low" need. Therefore fuzzy logic allows us to model this type of knowledge [9]. We have defined a fuzzy decision system in which the degree of necessity to check the information is evaluated from two variables, the user reliability and the number of contributions. The variables have been modeled with the following labels:

- Reliability: minimum, low, normal and high.

- Number of contributions: very few, few, average, many

- Checking_degree: null, low, normal, high.

Figure 4 shows how these variables have been modeled. Drone-like agents apply these rules in order to determine if the intervention of an expert is necessary and reports to the swarm with the results.

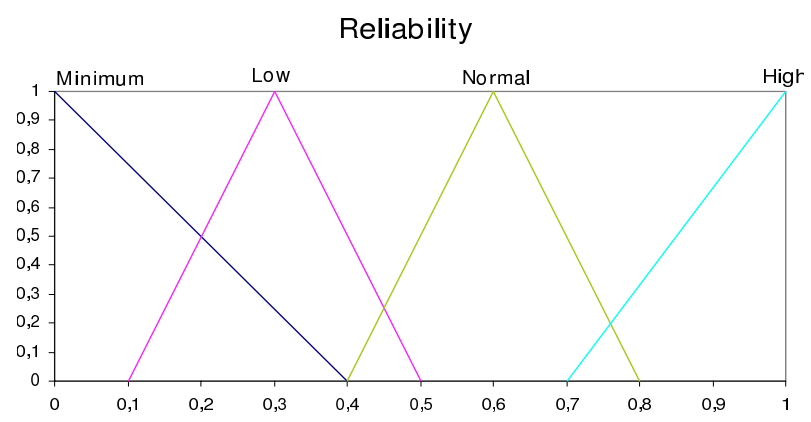

Fig. 4. Reliability fuzzy variables for deriving the degree of necessity to check information.

\subsection{User's reliability update}

Confidence of the knowledge stored in the hive depends a lot on the users who interact with the system, all of whom have different backgrounds and thus dif- 
ferent reliability degrees. For this reason, a mechanism to control and adapt the users' reliability according to their contributions is required. In particular, a user providing correct contributions should be rewarded by increasing their reliability; conversely, when a user provides incorrect contributions, their reliability should be decreased.

Defining correct contributions is somewhat difficult in terms of our application domain, that of traditional knowledge, in which the knowledge is never absolutely certain. However, we have defined this term on the basis of the opinion of the majority of users. Then, contributions are considered correct if they coincide with the current value of the corresponding knowledge to which they are contributing.

Then, a single user can be involved in different contributions, with different correctness values. Sometimes contributions are correct, sometimes they are not. So, to some extend, the user has shown a certain degree of coincidence between his/her contributions and the knowledge stored in the system. We model the degree of coincidence as follows:

$$
\text { hit_degree }=\frac{\text { hit_contributions }}{\text { new_contributions }}
$$

Where hit_contributions is the number of correct contributions, while new_ contributions is the total number of contributions that the user has provided since the last reliability update. The hit_degree is 1 when all the user's contributions coincide with the rest of the users, however, this is not a typical situation and normally the hit_degree is under 1.

Then, the hit_degree of the user is compared with the total number of new contributions to the system, and a change in the reliability degree is computed. This certainty degree is once again an imprecise measure and we have used fuzzy logic to model it. On this occasion, input variables are hit degrees and system_contributions, while the outcome of the fuzzy system is the change_degree.

- Hit_degree: minimum, low, normal, high

- System_contributions: very few, few, average, many.

- change_degree: low, no_touch, high.

Quality forager-like agents are responsible for updating the user's reliability according to the results of the fuzzy system.

\section{Experiments and results}

The system En_C_Prou has been implemented in JADE, deploying Apache for the web service, using PHP and MYSQL for dealing with the database. In the user interface, there us a left menu that allows the user to query some information, to upload new information, and so on. If the user chooses "query", then the system shows all the information regarding a subject. When the user action is "upload", then the system prompts the user with the current attribute list, the hit can link with either existing or new concepts. The current prototype is 
accessible at the following URL: http://xixi.udg.es, it works in an experimental way. Current users are volunteers of our lab who are external to the system development.

With regards to the different methods proposed, we first studied the evolution dynamics of the confidence values of the knowledge (see figure 5) based on system simulations. Experiments were carried out with different numbers of contributions in the same time instant, rating from 0 to 6 , different confidence values (from -0.9 to 0.9 ), and up to 20 evolving times. The behavior of the system shows that the confidence remains stable when the user's reliability is high (around 0.9).

Second, we analyzed the method proposed to determined the degree of necessity to check the information. As shown in Figure 6 (left) the system sustains a balanced behavior regarding the amount of user's contributions and their reliability. A user with a low reliability and with many contributions generates a need for significant checking. While a user with a high reliability, with many contributions generates a moderate need for checking.

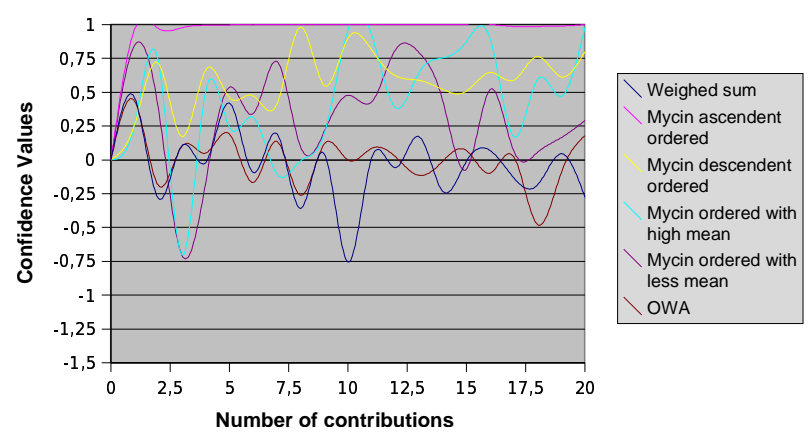

Fig. 5. Evolution dynamics of the confidence values.

Finally, the reliability updating method has also been analyzed experimentally with different hit degrees and contribution amounts (see Figure 6 right). The system has also shown balanced behavior depending on the hit contributions of the user and the amount of contributions in the system. A user with a high hit degree and many contributions is likely to have his/her reliability increased. Conversely, the reliability of a user with a low hit degree is seen as defective, and so will have his/her reliability lowered. These results correspond to the last methods we have been testing in the system [14].

Most of the experiments performed, however, are based on laboratory contributions and simulations. In order to get more appropriate conclusions we need to check En_C_Prou with a lot more users. Particularly, we are in contact with a public institution that is responsible of the public libraries around the local area. Our purpose is to use his infrastructure to deliver the system, so that elderly people can use them. 

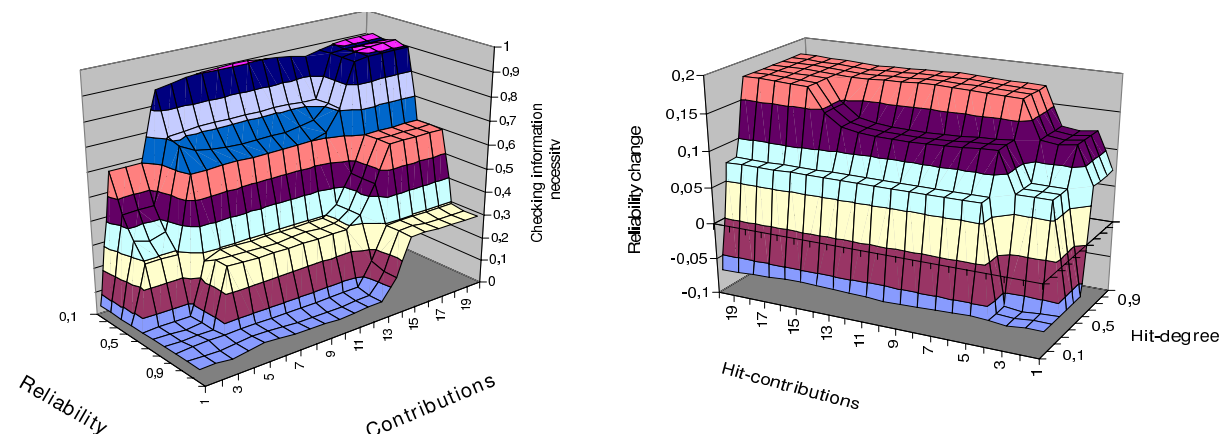

Fig. 6. Left: Results on the checking information necessity degree for several user's reliabilities and contributions. Right: Results ont the hit_degree analysis.

\section{Related work}

There are several previous works related to biologically inspired multi-agent systems, and particularly regarding swarm intelligence. Parunak in [16] shows interesting properties of ant-like agents, as for example the idea of keeping agents small. Bonabeau, Dorigo and Thereaulaz [2] is also a good reference for applications based on such kind of swarm inspired systems. There is also an increasing interest on applying such kind of systems to deploy industrial applications. [6] and [12] are good examples about using swarm technology to modelling factory operations subjected to dynamic environment characteristics. Particularly, Hadeli and their colleagues [6] show how swarm intelligence provides reliability and reactivity to the systems without the need of a central coordination mechanism.

Regarding the specific domain of information gathering, [7] has also used the paradigm of swarm intelligence to explore and exploite web information. Such kind of exploration techniques have also been used to automatically build ontologies, as for example in [11]. We think that such kind of approaches are complementary to ours. So we are not exploring but analyzing the information that users are uploaded to our web with a given purpose. The work of Agassounon, regarding optimizating information retrieval can also be clustered in this kind of exploratory facet of information that differs from our approach.

A more closer approach is the one developed by Yang and Kamel [18]. They propose the use of different swarms so that each swarm learn a different cluster, and the ensemble of all of them results in a more efficient clustering. We believe that such approach can be useful in our system in a future work, so we can build several swarms according to different user communities (for example, geographically distributed) and finally combine the results.

Regarding the particularly properties of swarm-like algorithms, we will like to compare here our particular implementation for Cultural Heritage, and the approaches followed by Cultural algorithms. Cultural algorithms are a specific form of evolutionary computation that utilizes culture as a vehicle for storing 
relevant information that is accessible to all members of the population over the course of many generations $[12,8]$. The different steps of the algorithm comprises an evaluation and voting scheme, that we can compare to our knowledge inference mechanism, in which the degree of coincidence among users is taken into account for knowledge validation. We are in some sense surprising to find such similarities with our system and Cultural algorithm, being both kinds of systems developed for so different purposes.

Finally, there is a collaborative approach in the Internet for gathering information that is called wikipedia (http://www.wikipedia.org/). This is an on-line encyclopedia written by hundreds of volunteers. The computer tool that supports it is a simple edition program called Wiki, which follows the free code style. One of the most original elements of the wiki is that entries can be commented on and broadened by other users. Everybody is free to propose new definitions of terms. The edition is totally controlled by the user. Everybody can modify the definitions without any limitation, except for the prerequisite of being registered. Our proposal differs from the wikipedies since the information entered by users can only be modified by agents acting inside the system and, consequently, by the confidence and reliability methods deployed as well as the self-organization (resemblance, ties) of the information.

\section{Conclusions}

Traditional knowledge is a patrimony that we cannot permit ourselves to lose. The importance of preserving the knowledge of elderly people has motivated the development of our En_C_Prou system. We have shown throughout the paper how we can model a swarm of bee-like agents that gather the information of non technologically skilled users. Each bee-like agent performs its individual task of processing the information, so finally, a repository of traditional knowledge is achieved.

In future work we propose broadening the experimentation by installing the system in more powerful equipment. Consequently, it will be necessary to evaluate the evolution of the system when applied to great volumes of data coming from out of the lab information sources.

\section{Acknowledgments}

This research project has been partially funded by the Spanish MEC project TIN2004-06354-C02-02. Thanks to Eduard Muntaner and Maria de los Llanos Tena for their contribution in the system implementation.

\section{References}

1. En_c_prou. http://xixi.udg.es/.

2. E. Bonabeau, M. Dorigo, and G. Théraulaz. Swarm intelligence. from natural to artificial systems. Oxford University Press, 1999. 
3. Théraulaz G. Bonabeau, E. Swarm smarts. Scientific American, 2000.

4. Bernat Capó. Costumari valencià 2. Edicions el Bullent, 1994.

5. V.A. Cicirello and S. F Smith. Wasp-like agents for distributed factory coordination. In Autonomous Agents and Multi-Agent Systems, volume 8, pages 237-266, 2004.

6. K. Hadeli, P. Valckenaers, B. Saint-Germain, P. Verstraete, C. BalaZamfirescu, and H. Van Brussel. Emergent forecasting using a stigmergy approach in manufacturing coordination and control. In Proceedings ESOA Workshop, 2004.

7. S. Hassas. Using swarm intelligence for dynamic web content organizing. In Proc. Swarm Intelligence, 2003.

8. Radu Iacoban, R.G. Reynolds, and J. Brewster. Cultural swarms: Modeling the impact of culture in social interactions and problem solving. In Proc. Swarm Intelligence, 2003.

9. Folger T. Klir, G. A. fuzzy sets, uncertainly, and information. Prentice Hall, 1992.

10. J. Pellicer. Costumari botànic. Picanya: Edicions del Bullent SL., 2000.

11. D. Rianyo, A. Moreno, D. Isern, J. Bocio, D. Sànchez, and L. Jiménez. Knowledge exploitation from the web. In Proc. PAKM, 2004.

12. N. Rychtyckyj, D. Ostrowski, G. Schleis, and R.G. Reynolds. Using cultural algorithms in industry. In Proc. Swarm Intelligence, 2003.

13. E.H. Shortliffe. Computer based medical consultation: Mycin. Elsevier, New York, 1976.

14. M.LL. Tena. Manteniment de la fiabilitat dels usuaris i de la confianca en la informació d'uan base de coneixements populars autoorganitzada a través d'un sistema multiagent. In MsCThesis, University of Girona (In Catalan), 2004.

15. A. Valls Mateu. Clusdm: A multiple criteria decision making method for heterogeneus data sets. In PhDThesis, IIIA, CSIC, Bellaterra, Spain, 2003.

16. H. Van Dyke Parunak. Go to the ant: Engineering principles from natural multiagent systems. In Annals of Operations Research., volume 75, pages 69-101, 1997.

17. D. R. Wilson and T. R. Martinez. Improved heterogeneous distance functions. In Journal of Articial Intelligence Research, volume 6:1, pages 1-34, 1997.

18. Y. Yang and M. Kamel. Clustering ensemble using swarm intelligence. In Proc. Swarm Intelligence, 2003. 